\title{
What Effects Might Exenatide have on Non-Motor Symptoms in Parkinson's Disease: A Post Hoc Analysis
}

\author{
Dilan Athauda ${ }^{\mathrm{a}}$, Kate Maclagan ${ }^{\mathrm{b}}$, Natalia Budnik ${ }^{\mathrm{c}}$, Luca Zampedri ${ }^{\mathrm{c}}$, Steve Hibbert ${ }^{\mathrm{b}}$, \\ Simon S. Skene ${ }^{\mathrm{b}, \mathrm{d}}$, Kashfia Chowdhury ${ }^{\mathrm{b}}$, Iciar Aviles-Olmos ${ }^{\mathrm{a}}$, Patricia Limousin ${ }^{\mathrm{a}}$ \\ and Thomas Foltynie ${ }^{\mathrm{a}, *}$ \\ ${ }^{a}$ Sobell Department of Motor Neuroscience, UCL Institute of Neurology and The National Hospital for Neurology \\ and Neurosurgery, Queen Square, London, UK \\ ${ }^{\mathrm{b}}$ UCL Comprehensive Clinical Trials Unit (UCL CCTU) \\ ${ }^{\mathrm{c}}$ Leonard Wolfson Experimental Neuroscience Centre, London, UK \\ ${ }^{\mathrm{d}}$ University of Surrey, Surrey Clinical Trials Unit, UK
}

Accepted 27 March 2018

\begin{abstract}
.
Background: Exenatide is a GLP-1 receptor agonist that was recently studied for potential disease-modifying effects in a randomised, placebo-controlled clinical trial in patients with moderate stage Parkinson's disease, and showed positive effects on the motor severity of the disease which were sustained 12 weeks beyond the period of exenatide exposure. Analysis of pre-defined secondary outcomes revealed no statistically significant differences between patients treated with exenatide in total non-motor symptom burden and overall quality of life measures.

Objective: The response of individual non-motor symptoms to an intervention may vary and thus this post hoc analysis was conducted to explore the possible effects of exenatide compared to placebo on individual non-motor symptoms.

Results: Compared to placebo, patients treated with exenatide-once weekly had greater improvements in individual domains assessing mood/depression across all observer-rated outcome measures after 48 weeks including the "mood/apathy" domain of the NMSS, -3.3 points $(95 \% \mathrm{CI}-6.2,-0.4), p=0.026$; the "mood" score (Q1.3+Q1.4 of the MDS-UPDRS Part 1$),-0.3$ points $(95 \% \mathrm{CI}-0.6,-0.1), p=0.034$; and a trend in the MADRS total score, -1.7 points $(95 \% \mathrm{CI}-3.6,0.2), p=0.071$. In addition, there was an improvement in the "emotional well-being" domain of the PDQ-39 of 5.7 points ((95\%CI -11.3, -0.1$)$, $p=0.047$ though these improvements were not sustained 12 weeks after exenatide withdrawal. At 48 weeks these changes were of a magnitude that would be subjectively meaningful to patients and were not associated with changes in motor severity or other factors, suggesting exenatide may exert independent effects on mood dysfunction.

Conclusions: These exploratory findings will contribute to the design of future trials to confirm the extent of motor and non-motor symptom effects of exenatide in larger cohorts of patients.
\end{abstract}

Keywords: Parkinson's disease, exenatide, non-motor symptoms, clinical trial, GLP-1 agonist

\section{INTRODUCTION}

*Correspondence to: Professor Thomas Foltynie, Sobell Department of Motor Neuroscience, UCL Institute of Neurology and The National Hospital for Neurology and Neurosurgery, Queen Square, London, WC1N 3BG, UK. Tel.: +44 203448 8726; Fax: +44 203448 8642; E-mail: T.Foltynie@ucl.ac.uk.
The importance of non-motor symptoms in Parkinson's disease (PD) is increasingly recognised. Non-motor symptoms such as depression and REM sleep behaviour disorder can often precede the 
appearance of motor deficits, while others such as cognitive impairment and autonomic dysfunction can come to dominate the clinical picture in advancing stages and influence the need for advanced care [1]. The pathology underlying the appearance of non-motor symptoms is uncertain but is thought to encompass the progressive degeneration of dopaminergic neurons together with accumulating noradrenergic and cholinergic deficits in the central and peripheral nervous system causing an array of symptoms including depression, apathy, cognitive impairment, sleep disorders and sensory symptoms. These features can have a greater impact on healthrelated quality of life measures than the motor deficits [2]. Non-motor symptoms can also be influenced by gender, motor severity, age and to a variable extent by levodopa [3] but in contrast to the number of effective dopamine-replacement symptomatic therapies for motor symptoms, treatment of non-motor symptoms is often limited and unsatisfactory. Thus, as non-motor symptoms can be significant, often prominent factors in determining quality of life, any potential novel therapeutic aimed at slowing disease progression must not only target dopaminergic pathways but also influence non-dopaminergic targets.

Exenatide was the first synthetic GLP-1 receptor agonist to be developed for the treatment of Type 2 diabetes [4] and was recently studied for potential disease-modifying effects in a randomised, placebocontrolled clinical trial in patients with moderate stage Parkinson's disease, showing positive effects on the motor severity of the disease (measured after overnight dopaminergic medication withdrawal), which were sustained 12 weeks beyond the period of exenatide exposure [5]. Analysis of pre-defined secondary outcomes revealed no statistically significant differences between patients treated with exenatide in total non-motor symptom burden as assessed by the non-motor symptom scale (NMSS), MDS-UPDRS Part 1, Montgomery-Asberg depression rating scale (MADRS) and Mattis Dementia rating scale and also in general health-related quality of life assessments including the Parkinson's disease questionnaire (PDQ-39), EQ5D index and EQD-VAS scales.

Although these scales are important in recognising the overall impact of non-motor disturbances, often they are too imprecise in assessing specific non-motor symptom pathophysiology [6] and the response of individual non-motor symptoms to an intervention may vary, due to the various circuits involved and the variability of severity of each symptom subdomain in patients of different disease stages [7, 8]. The trial included a heterogeneous cohort of patients suffering from a variety of non-motor symptoms and it is possible that potentially useful effects on individual non-motor symptoms may have been obscured by the imprecision associated with total scale scores. Given the importance of non-motor symptoms in predicting quality of life, this post hoc analysis of the ExenatidePD trial was conducted to explore possible effects of exenatide compared to placebo on individual nonmotor symptoms utilising subdomain scores across the NMSS, EQ5D, PDQ-39, MDS-UPDRS Part 1, MADRS and Mattis DRS-2 and thus inform on planning which secondary outcomes might be included in future trials of exenatide as a potential disease modifying treatment.

\section{METHODS}

The Exenatide-PD study was a randomised, double-blind, placebo controlled trial of exenatide $2 \mathrm{mg}$ once-weekly in Parkinson's disease assessing the effects of this drug on disease progression over 60 weeks, the details of which have been published previously [5]. The trial had a washout design, comprising 60 patients randomised to self-inject either exenatide $2 \mathrm{mg}(n=31)$ or placebo $(n=29)$ onceweekly for 48 weeks, followed by drug withdrawal and a final visit 12 weeks later. At each visit, patients were asked to attend after an overnight withdrawal from Parkinson's disease medication, and were assessed using the Movement Disorders Society Unified Parkinson's Disease Rating Scale (MDSUPDRS). Patients were asked to take their usual medication and a variety of observer- and self-rated assessments to evaluate non-motor symptoms were undertaken in the "on" state.

\section{Non-motor outcomes assessment scales}

The NMSS is a 30 -item clinician-rated scale designed to assess the severity and frequency of non-motor symptoms over the last month and is sub-divided into nine domains: cardiovascular; sleep/fatigue; mood/apathy; perceptual problems /hallucination; attention/memory; gastrointestinal tract; urinary; sexual function; and miscellaneous [9].

The MDS-UPDRS Part 1 is a two-part questionnaire designed to evaluate non-motor symptom burden and comprises part 1A, 6 questions rated by the observer that focuses on neuropsychiatric symptoms and part 1B, 5 questions composed of miscellaneous non-motor symptoms self-completed by 
the patient [10]. Each item is rated on a scale of 0-4 and correlates strongly with the NMSS [11]. For these analyses, we subdivided the total scores into sub scores for mood dysfunction (Q1.3-Q1.4), apathy (Q1.5), impulsive compulsive behaviour (Q1.6), sleep (Q1.7-1.8) and autonomic dysfunction: urinary (Q1.10), constipation (Q1.11) and cardiovascular symptoms (Q1.12).

The MADRS is 10-item, observer-rated depression rating scale that due to less reliance on somatic symptoms, is particularly useful in screening and measuring the severity of depression in PD and has been used previously to assess response to antidepressant medication [12].

The Mattis Dementia Rating scale (Mattis-DRS) is widely used to screen dementia and track changes in cognitive performance. It provides an overall score of cognitive function (total score 0-144) and is based on performance from five subscales: attention, initiation, construction, conceptualization and memory. For our analysis, patients with a score of $<137$ were defined as mild cognitive impairment [13].

The PDQ-39 is a 39-item questionnaire reporting the quality of life determining aspects of PD over the last month as perceived and interpreted by the patient. It is widely used as an outcome measure in clinical trials. The 39 questions are divided into 8 dimensions: mobility (Q1-Q10), activities of daily living (ADL) (Q11-Q16), emotional well-being (Q17-Q22), stigma (Q23-Q26), social support(Q27-Q29), cognition (Q30-Q33), communication(Q34-Q36), and bodily discomfort(Q37-Q39) allowing individual index scores for these domains to be calculated [14].

The EQ-5D is a self-assessment questionnaire that measures broad health-related quality of life and has been used to capture disease burden in PD patients [15]. It comprises five questions on mobility, self-care, usual activities and psychological state, allowing a summary index score of 0-1 to be generated, with the maximum score of 1 indicating best health. In addition, there is a visual analogue scale (VAS) marked 0-100 which is self-completed on the day to indicate a general health status, with 100 indicating the best health status.

\section{Assessment of individual non-motor symptoms}

Non-motor symptoms were grouped into "neuropsychiatric" (mood, cognition, apathy, hallucinations, ICB), "autonomic" (constipation, urinary, cardiovascular, sexual function), and "other" (sleep, fatigue) domains using individual subdomain scores across the NMSS, MDS-UPDPRS Part 1, MADRS and Mattis-DRS-2 rating scales (Table 1).

\section{Statistical analyses}

The aim of these exploratory analyses was to evaluate the impact of treatment allocation (exenatide or placebo) on individual non-motor symptoms as assessed by the difference between the individual domains of the NMSS, PDQ-39, MDS-UPDRS Part 1, MADRS scores at 48 and 60 weeks follow up. The analysis used a regression (ANCOVA, analysis of co-variance) approach to adjust for known confounders including age, gender, baseline motor severity, change in levodopa equivalent dose and baseline non-motor assessment values, (i.e., beyond those confounders adjusted for in the original pre-defined statistical analysis plan). To ascertain the effects of treatment group on the presence/absence of depression, apathy and MCI, logistic regression models were constructed to ascertain the effects of exenatide on the likelihood that participants reported these symptoms at 48 weeks, using the baseline presence of these non-motor symptoms as covariates.

The study is a post hoc analysis of the effects of exenatide on individual non-motor symptoms and therefore all $p$ values presented for these outcome measures are purely exploratory and do not infer statistical significance. All study analyses were performed using STATA/MP (StataCorp, Version 13.0, College Station, TX, USA) and SPSS (IBM, Version 21.0. Armonk, NY: IBM Corp). Data represent the mean and standard error of the mean.

\section{RESULTS}

\section{Patient characteristics}

As previously reported, patient demographics and baseline characteristics were generally similar between the two randomly allocated groups though the exenatide group were slightly older and had higher baseline MDS-UPDRS part 3 scores and slightly lower Levodopa Equivalent dose (LED (Athauda et al. 2017)). Total nonmotor symptom burden at baseline (assessed by total NMSS) was slightly lower in the exenatide group than among those assigned to placebo, while MDS-UPDRS Part 1 scores were slightly higher. 
Table 1

Non-motor symptoms as grouped by individual subdomain scores

\begin{tabular}{|c|c|c|}
\hline Non-motor Feature & Symptom & Outcome measure \\
\hline \multirow[t]{5}{*}{ Neuropsychiatric } & Mood & $\begin{array}{l}\text { "Mood" domain of NMSS; "Emotional well-being" of } \\
\text { PDQ-39; proportion of patients reporting depressive } \\
\text { symptoms (MADRS); Q1.3 + Q1.4 of MDS-UPDRS } \\
\text { Part I }\end{array}$ \\
\hline & Cognition & $\begin{array}{l}\text { "Attention/memory" of the NMSS; "Cognition" domain } \\
\text { of the PDQ-39; individual domains of the } \\
\text { Mattis-DRS-2; the proportion of patients with MCI at } \\
48 \text { weeks; Q1.1 of the MDS-UPDRS Part I }\end{array}$ \\
\hline & Apathy & Q1.5 of MDS-UPDRS Part I; \\
\hline & Hallucinations & $\begin{array}{l}\text { "Hallucinations" domain of the NMSS; Q1.2 of the } \\
\text { MDS-UPDRS Part I; }\end{array}$ \\
\hline & Impulsive compulsive behaviours & Q1.6" of the MDS-UPDRS Part I \\
\hline \multirow[t]{2}{*}{ Autonomic } & Constipation, urinary symptoms & $\begin{array}{l}\text { "Urinary" and "GI tract" domains of the NMSS; } \\
\text { Q1.10+1.11 }\end{array}$ \\
\hline & Cardiovascular & $\begin{array}{l}\text { "Cardiovascular/falls" domain of the NMSS; Q1.12 of } \\
\text { MDS-UPDRS Part II; changes related to systolic } \\
\text { diastolic BP }\end{array}$ \\
\hline Sleep & Sleep & $\begin{array}{l}\text { "Sleep" domain of the NMSS; Q1.7+1.8 of } \\
\text { MDS-UPDRS Part II, }\end{array}$ \\
\hline Sensory & Pain & $\begin{array}{l}\text { "Bodily discomfort" of the PDQ-39; Q1.9 of } \\
\text { MDS-UPDRS Part II; }\end{array}$ \\
\hline Other & Fatigue & Q1.13 of the MDS-UPDRS Part II; \\
\hline
\end{tabular}

\section{Effects of exenatide on overall quality of life}

In the previously reported, pre-defined comparison of health- related quality of life measures in patients treated with exenatide compared to placebo as measured by the PDQ-39 summary index, EQ3L$5 \mathrm{D}$ index and EQ-VAS scores, none of the differences reached the threshold for statistical significance. In this post-hoc analysis, at 48 weeks, patients in the placebo group declined by 0.03 (SEM1.4) points on the total PDQ-39 while patients in the exenatide group improved by 2.2 (SEM1.3) points a nonsignificant difference favouring exenatide of -2.2 points $(95 \% \mathrm{CI}-6.2,1.8), p=0.271$ while at 60 weeks patients in the placebo group declined by 1.1 (SEM 1.8) points and the exenatide group improved by 2.3 (SEM1.6) points, conferring a non-significant improvement of -3.5 points $(95 \% \mathrm{CI}-8.4,1.5)$, $p=0.166$.

\section{Post hoc analysis of effects of exenatide on Non-motor symptom subdomains}

\section{Mood}

There were strong correlations between individual domains scores assessing emotional dysfunction/depression across the NMSS, MDS-UPDRS Part 1 and MADRS. There was a strong correlation between the "mood/apathy" domain of NMSS and the "mood" score of MDS-UPDRS
Part 1 (rho $=0.692, p<0.0001)$, and the MADRS total score $(r h o=0.454, p=0.0001)$. At baseline, the burden of total non-motor symptoms in the NMSS was primarily driven by "mood/apathy" (rho $=0.609, p=<0.001)$ and "sleep" (rho $=0.602$, $p<0.001)$, and similarly for the total MDS-UPDRS Part 1 scores at baseline, the strongest correlations were observed for questions regarding depression (rho $=0.674, p=<0.001)$ and anxiety $($ rho $=0.633$, $p=<0.001$ ).

Compared to placebo, patients treated with exenatide-once weekly had numerically greater improvements in individual domains assessing mood/depression across all observer-rated outcome measures after 48 weeks including the "mood/apathy" domain of the NMSS, -3.3 points (95\% CI $-6.2,-0.4), p=0.026$; the "mood" score (Q1.3+Q1.4 of the MDS-UPDRS Part 1), -0.3 points (95\%CI $-0.6,-0.1), p=0.034$; and MADRS total score, -1.7 points $(95 \% \mathrm{CI}-3.6,0.2), p=0.071$ (Table 2 and Fig. 1). In addition, the proportion of patients reporting depressive symptoms (as defined by the total MADRS score $>7$ ) in the placebo group increased from $17 \%$ at baseline to $25 \%$ at 48 weeks while in the exenatide group the proportion of patients reporting depressive symptoms reduced from $23 \%$ at baseline to $6 \%$ of patients at 48 weeks. A binominal logistic regression was performed to ascertain the effects of exenatide on the likelihood that participants reported depressive 


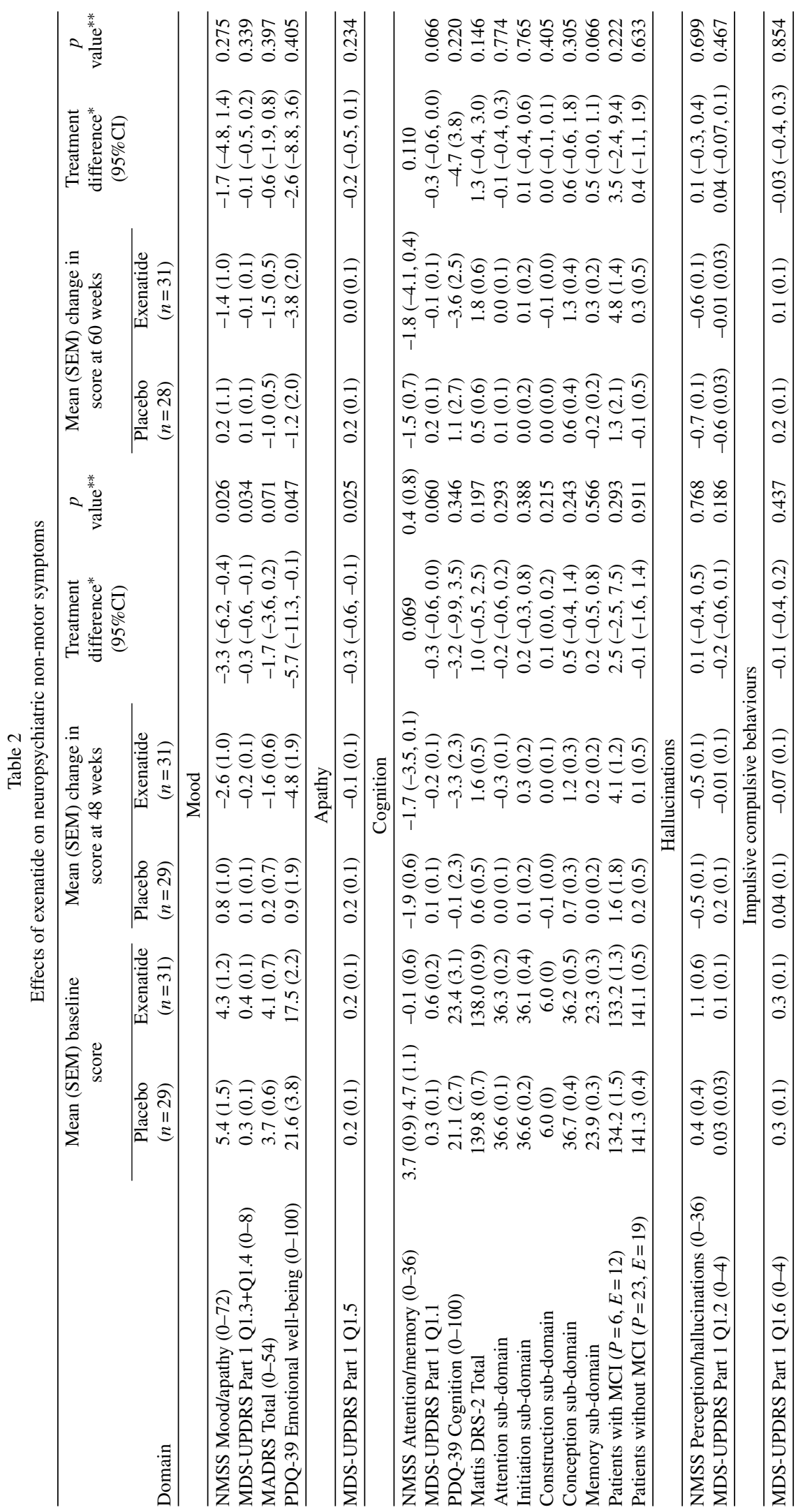




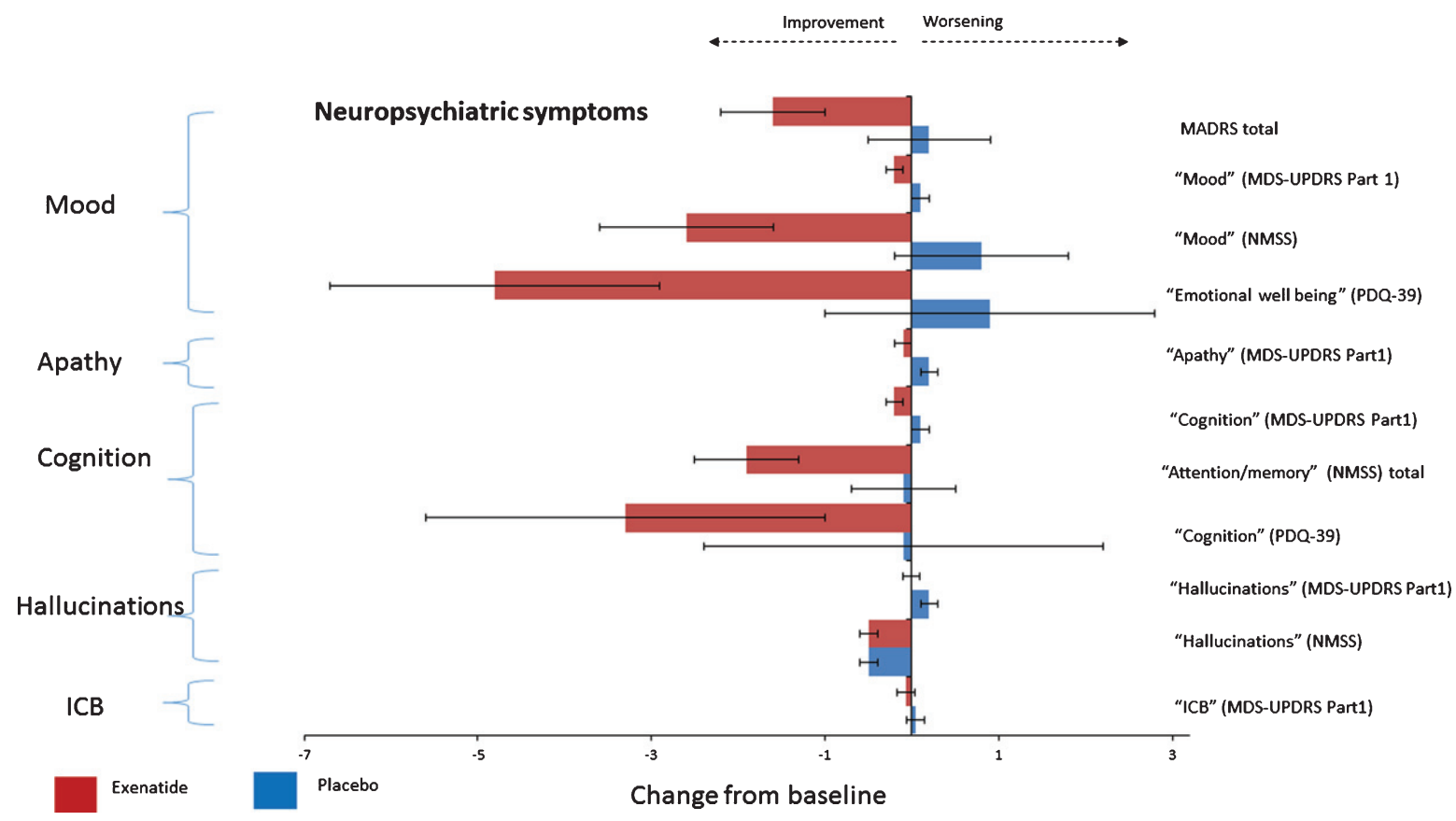

Fig. 1. Change from baseline on neuropsychiatric non-motor symptoms at 48 weeks.

symptoms at 48 weeks. In the logistic regression model, treatment allocation explained $23.0 \%$ of variance, $\chi 2(2)=8.36, p=0.015$ and correctly identified $84.4 \%$ of cases. Patients treated with exenatide had 4.8 times (95\% CI 26.3 to 0.88 ) lower odds of exhibiting depression than patients in the placebo group $(p=0.070)$.

Based on self-rating assessments, patients treated with exenatide reported improvement in the "emotional well-being" domain of the PDQ-39 of 4.8 points (SEM 1.9) compared to a decline in the placebo group of 0.9 points (SEM 1.9) at 48 weeks, with the resulting difference between groups of 5.7 points ((95\%CI $-11.3,-0.1), p=0.047)$ (Fig. 3). There were no significant differences in domains assessing mood at 60 weeks (Table 2). There was no correlation between improvements in emotional dysfunction/depression subdomains and changes in motor severity as assessed by the MDS-UPDRS Part 3 scores.

\section{Apathy}

At 48 weeks, patients treated with exenatide had numerically greater improvements in apathy as assessed by Q1 of the MDS-UPDRS Part 1, with a difference between groups of -0.3 points $(95 \% \mathrm{CI}-0.6$, -0.1 ), $p=0.025$ (Table 2 and Fig. 1). In the placebo group, the percentage of patients reporting apathy (score $>0$ ) increased from $10 \%$ at baseline to $28 \%$ at 48 weeks while in the exenatide group the percentage of patients reporting apathy reduced from $13 \%$ at baseline to $9 \%$ at 48 weeks. In the logistic regression model, treatment allocation explained $26.5 \%$ of variance in apathy, $\chi^{2}(2)=10.67, p=0.005$ and correctly identified $83.3 \%$ of cases. Patients treated with exenatide had 5.2 times (95\% CI 26.8 to 0.95 ) lower odds of exhibiting apathy than patients in the placebo group $(p=0.056)$. At the 60 week timepoint, there were no significant differences between the two groups.

\section{Cognition}

In the previously reported comparison of the total Mattis DRS-2 score in patients treated with exenatide, there was a difference favouring exenatide of 1.0 point $(95 \% \mathrm{CI}-0.5,2.5), p=0.197$. There was no evidence of changes in individual domain scores regarding attention, initiation, construction, conception and memory. Compared to placebo, patients treated with exenatide again had non-significant improvements in domains assessing cognition in the NMSS of -1.7 points $(95 \% \mathrm{CI}-3.5,0.1, p=0.069)$ and Q1.1 of MDS-UPDRS Part 1 of -0.3 points $(95 \% \mathrm{CI}-0.6,0.0, p=0.060)$. 


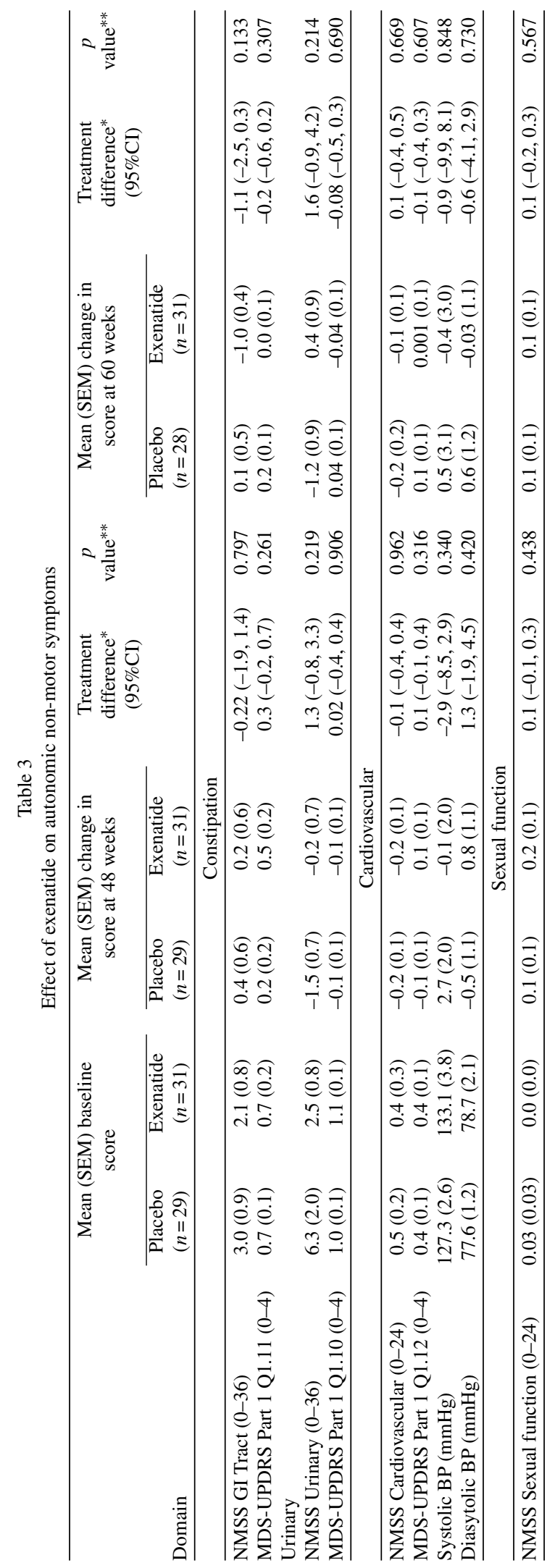




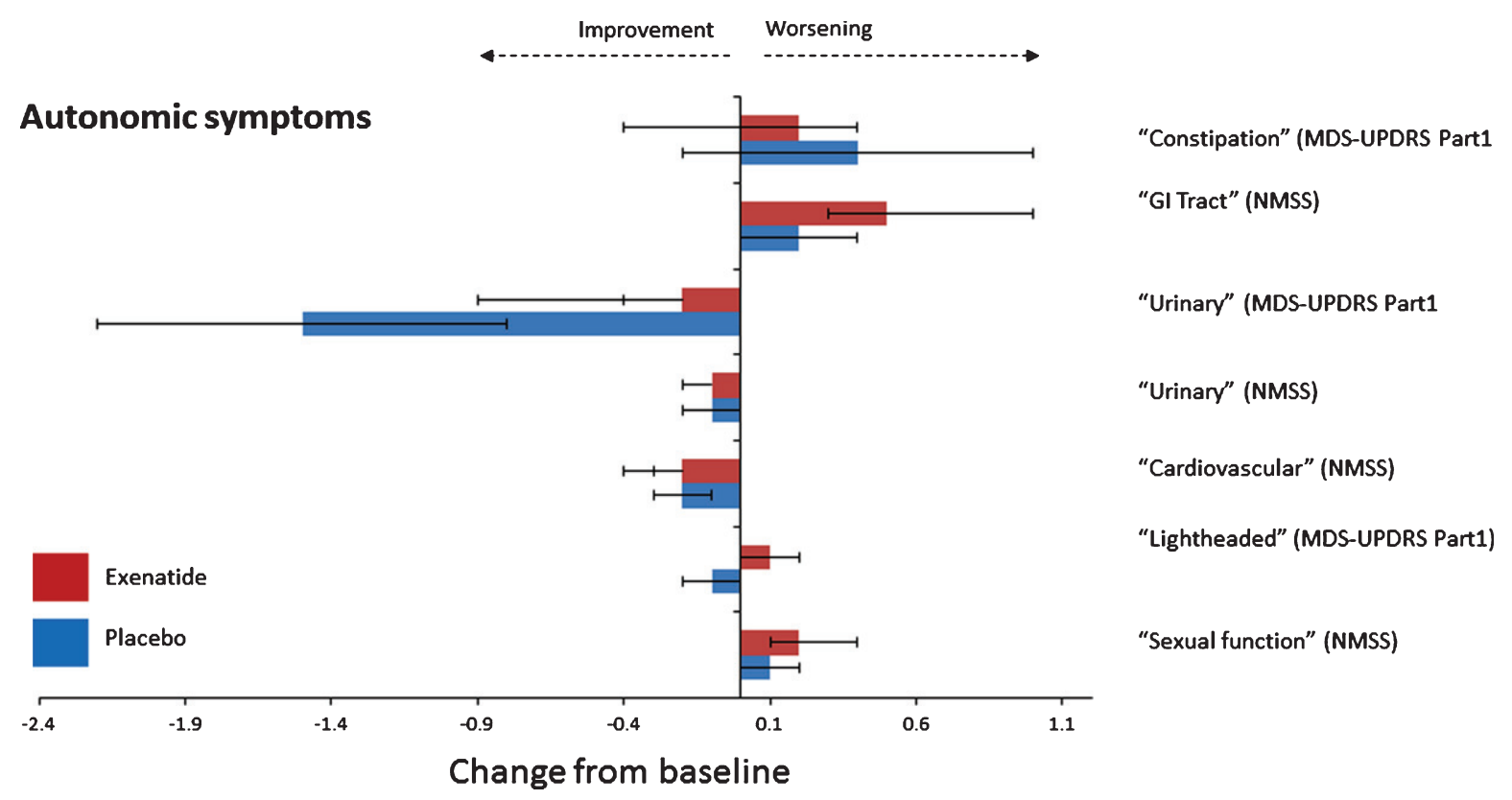

Fig. 2. Change from baseline on autonomic non-motor symptoms at 48 weeks.

While there was no change in the percentage of patients with MCI (defined as Mattis-DRS2 score $<137)$ in the placebo group at 48 weeks $(21 \%$ vs $21 \%$ at baseline), in the exenatide group the percentage of patients with MCI reduced from $39 \%$ at baseline to $16 \%$ at 48 weeks. In the logistic regression, treatment allocation predicted $18.9 \%$ of the variance of MCI $\chi^{2}(2)=6.132, p=0.047$, and correctly identified $90 \%$ of cases of MCI. Patients treated with exenatide were associated with a 3.7 times (95\%CI 27.0 to 0.51 ) lower likelihood of exhibiting MCI at 48 weeks compared to placebo $(p=0.198)$.

\section{Hallucinations/Impulsive compulsive behaviours}

There were no consistent effects of exenatide on hallucinations as assessed by the NMSS domain "perception/hallucinations" or MDS-UPDRS Part 1 Q1.2 at 48 or 60 week time points.

\section{Autonomic non-motor symptoms}

There was no consistent direction of effects of exenatide on observer or self-assessed domains regarding gastrointestinal, urinary cardiovascular symptoms or sexual function as assessed by the "Gastrointestinal", "Urinary," "Cardiovascular" and "Sexual Function" domains of the NMSS and MDS-UPDRS Part 1 Q1.0 and Q1.11 and Q1.12 (Table 3 and Fig. 2) at 48 or 60 weeks. There was no change in BP at the end of 48 weeks. Patients in the placebo group had an increase in systolic BP of $2.7 \mathrm{mmHg}$ compared to a reduction of $0.1 \mathrm{mmHg}$ in the exenatide group, translating to a difference in systolic BP of $2.9 \mathrm{mmHg}$ $(95 \% \mathrm{CI}-8.5,2.9, p=0.340)$. There was a marginal increase in diastolic BP of $1.3 \mathrm{mmHg}(95 \% \mathrm{CI}-1.9$, 4.5 ) in the exenatide group at 48 weeks in comparison to the placebo group.

\section{"Other" non-motor symptoms}

There were no significant differences between the two groups regarding domains assessing sleep. Compared to placebo, exenatide treated patients had a reduction of the "sleep/fatigue" subdomain score of the NMSS, -1.5 points $(95 \% \mathrm{CI}-4.0,1.0), p=0.274$ and "Sleep" of the MDS-UPDRS Part 1 (Q1.7, Q1.8), -0.1 points $(95 \% \mathrm{CI}-0.5,0.3, p=0.610)$ (Table 4$)$.

\section{Effects of exenatide on "Quality of life subdomains"}

Within the individual Quality of life subdomain scores, the greatest difference between exenatide treated patients and the placebo group was in the "emotional-well-being," subdomain at 48 weeks. There were no significant differences in other domains (Table 5). 
Table 4

Effects of exenatide on "Other" non-motor symptoms

\begin{tabular}{|c|c|c|c|c|c|c|c|c|c|c|}
\hline \multirow[b]{2}{*}{ Domain } & \multicolumn{2}{|c|}{$\begin{array}{c}\text { Mean (SEM) baseline } \\
\text { score }\end{array}$} & \multicolumn{2}{|c|}{$\begin{array}{l}\text { Mean (SEM) change in } \\
\text { score at } 48 \text { weeks }\end{array}$} & \multirow{2}{*}{$\begin{array}{c}\text { Treatment } \\
\text { difference* } \\
(95 \% \mathrm{CI})\end{array}$} & \multirow{2}{*}{$\begin{array}{c}p \\
\text { value }^{* *}\end{array}$} & \multicolumn{2}{|c|}{$\begin{array}{l}\text { Mean (SEM) change in } \\
\text { score at } 60 \text { weeks }\end{array}$} & \multirow{2}{*}{$\begin{array}{r}\text { Treatment } \\
\text { difference* } \\
(95 \% \mathrm{CI})\end{array}$} & \multirow{2}{*}{$\begin{array}{c}p \\
\text { value }^{* *}\end{array}$} \\
\hline & $\begin{array}{l}\text { Placebo } \\
(n=29)\end{array}$ & $\begin{array}{c}\text { Exenatide } \\
(n=31)\end{array}$ & $\begin{array}{l}\text { Placebo } \\
(n=29)\end{array}$ & $\begin{array}{c}\text { Exenatide } \\
(n=31)\end{array}$ & & & $\begin{array}{l}\text { Placebo } \\
(n=28)\end{array}$ & $\begin{array}{c}\text { Exenatide } \\
(n=31)\end{array}$ & & \\
\hline & & & & Sleep & & & & & & \\
\hline $\begin{array}{l}\text { NMSS } \\
\text { Sleep/fatigue } \\
(0-48)\end{array}$ & $7.6(1.2)$ & $5.5(1.0)$ & $-0.3(0.9)$ & $-1.8(0.9)$ & $\begin{array}{c}-1.5 \\
(-4.0,1.0)\end{array}$ & 0.274 & $1.0(1.1)$ & $-0.6(1.0)$ & $\begin{array}{c}-1.6 \\
(-4.9,1.6)\end{array}$ & 0.312 \\
\hline $\begin{array}{l}\text { MDS-UPDRS } \\
\text { Part 1 } \\
\text { Q1.7+Q1.8 } \\
\quad(0-8)\end{array}$ & $1.7(0.1)$ & $1.5(0.1)$ & $-0.2(0.1)$ & $-0.3(0.1)$ & $\begin{array}{c}-0.1 \\
(-0.5,0.3)\end{array}$ & 0.610 & $0.1(0.1)$ & $-0.2(0.1)$ & $\begin{array}{c}-0.3 \\
(-0.7,0.1)\end{array}$ & 0.090 \\
\hline
\end{tabular}

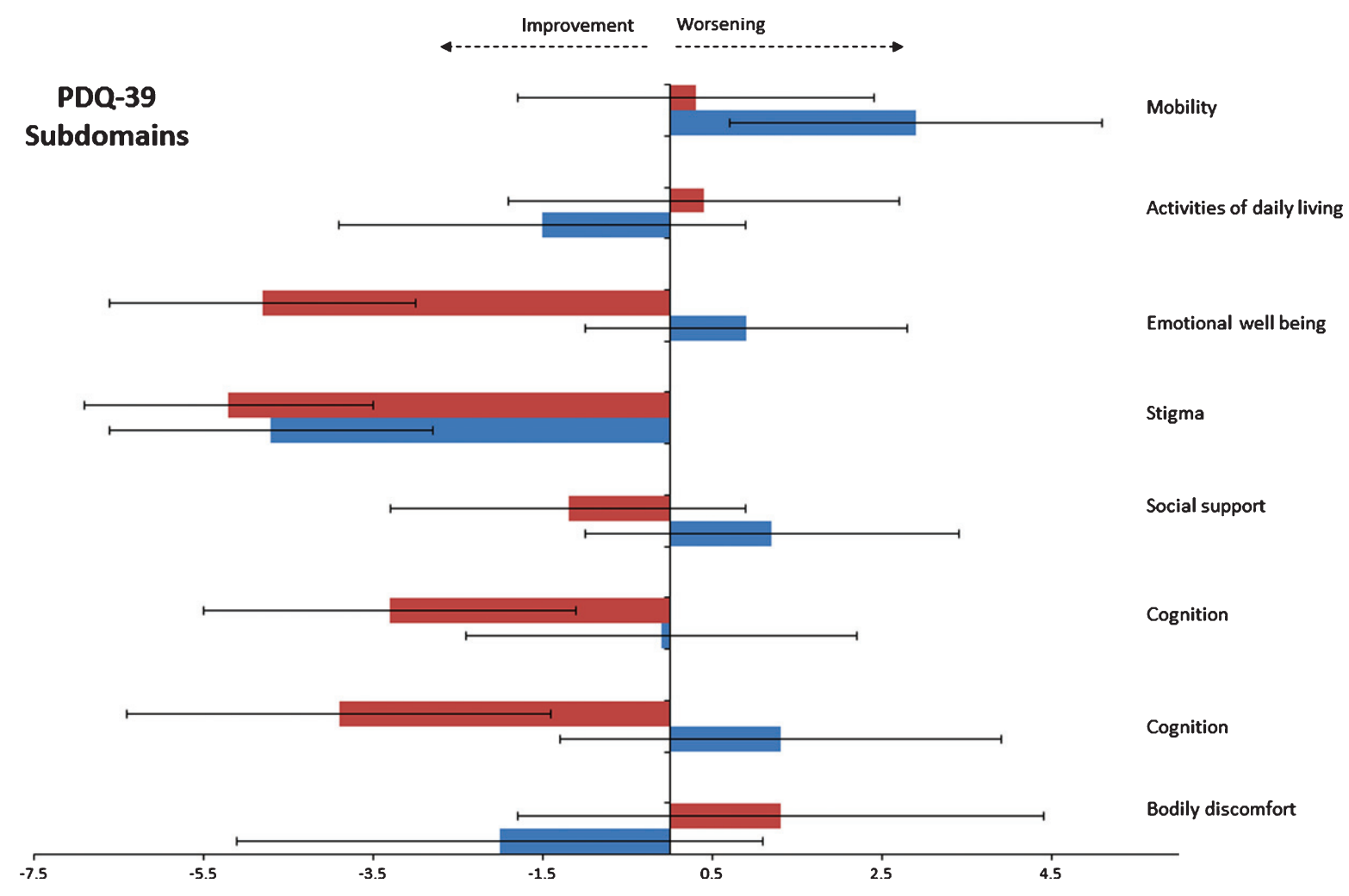

Fig. 3. Change from baseline on individual domains of the PDQ-39 at 48 weeks.

\section{DISCUSSION}

In this post hoc analysis of a previously reported randomised, placebo-controlled trial, there were consistent effects in exenatide-treated patients on individual domains assessing emotional dysfunction/depression domains as assessed by the NMSS, MDS-UPDRS Part 1 and MADRS at 48 weeks. Although this study was not powered to determine the effects of exenatide on non-motor symp- toms, and all the analyses were post-hoc, the advantages in exenatide-treated patients appeared consistent across a variety of observer and patientled scales assessing mood suggesting that further investigations of exenatide should include specific evaluation of mood dysfunction and its possible anti-depressant-like effects in patients with PD. Previous studies have demonstrated that a change of at least 2.62 points on the "mood/apathy" domain of the NMSS and 4.2 points on the "emotional 


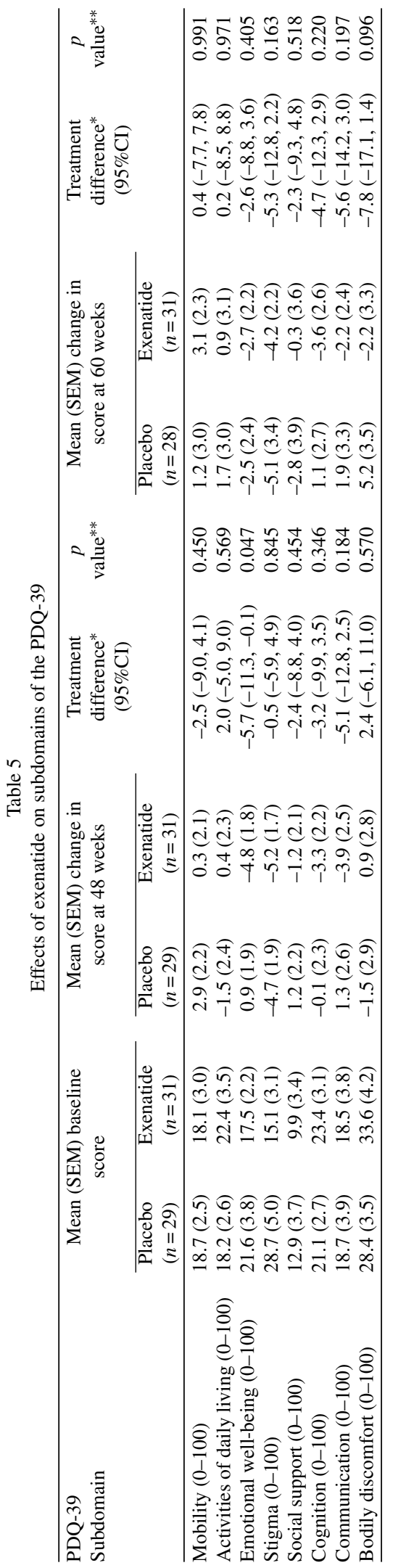

well-being" domain of the PDQ-39 constitutes the minimal clinically important differences, suggesting that if confirmed, these exenatide-conferred changes are in the range that would be meaningful to patients $[16,17]$. There was no correlation between improvements in emotional dysfunction/depression subdomains and changes in motor severity as assessed by the MDS-UPDRS Part 3 scores, suggesting that changes in these domains were not simply a result of motor improvements associated with exenatide treatment and may indicate that nondopaminergic and non-nigrostriatal mechanisms may be involved.

These results are in contrast to the comparison of the whole NMS scale, which did not detect differences between the groups possibly because of the lack of change in autonomic and sleep effects. Whether exenatide might have had greater effects on cognitive performance among a cohort of patients with more advanced disease in which the rate of cognitive decline is faster (as was observed in the first trial of exenatide in PD patients $[18,19]$ requires further study.

GLP-1 receptors are found throughout the frontal cortex, amygdala, dorsal raphe and hippocampus, areas all implicated in the pathophysiology of depression/emotional dysfunction in PD and central GLP-1 receptor stimulation has been demonstrated to affect serotonin signalling in the amygdala, widely held to be a neurotransmitter involved in mood [20]. Furthermore rodents chronically treated with exendin- 4 demonstrate significantly reduced depressive-like behaviour in a forced-swim test [21] and also demonstrated anxiolytic and anti-depressant-like effects in a lipopolysaccharide model of PD that were associated with alteration in dopaminergic signalling [22]. Given that by the 60 week time point, there were no significant differences in mood between the two groups, these potential non-motor symptom changes may be more likely related to neurochemical effects as a result of GLP-1 receptor stimulation rather than relating to the neurodegenerative process itself. However, non-motor fluctuation is less well understood than motor fluctuations and often non-motor symptoms do not correlate with motor fluctuation or with measures of disease progression, making longitudinal fluctuations difficult to interpret [23].

Apathy is a challenging non-motor symptom in $\mathrm{PD}$, affecting $17 \%-60 \%$ of patients with PD, and is regarded as an independent nosological enitity from depression or cognitive dysfunction with dis- 
tinct pharmacological targets [24]. While our post hoc analyses indicated a small advantage in apathy among the exenatide treated group, it is difficult to interpret the clinical relevance of these very small numerical improvements.

\section{Limitations}

Our post hoc analysis was performed to aid in future planning of exenatide trials in PD only and the results must therefore not be over-interpreted. Furthermore, despite randomisation, there were some differences between the two groups at baseline, with the placebo group being slightly younger with less motor severity and higher LED and nonidentical NMS severity. Although we have adjusted for these confounders in our analysis, they may have influenced non-motor symptom progression, in that patients with more severe PD have been reported to be more responsive to non-motor improvements [25] and dopaminergic medication can also influence certain non-motor symptoms [26].

The average disease duration of our cohort was 6.4 years, and previous studies have indicated that significant progression of non-motor symptoms and quality of life scores often occurs in the earliest stages and can then stabilise, however this is dependent on the specific non-motor symptom being studied as cognitive and autonomic features tend to be associated with later disease stages. Also, the sensitivity of the assessment of some non-motor symptoms using NMSS and MDS-UPDRS Part 1 may be suboptimal therefore determining whether exenatide had specific effects on sleep or apathy would be better performed using dedicated instruments such as sleep studies, and validated domain specific questionnaires.

\section{Conclusion}

In this post-hoc, exploratory analysis we have identified that exenatide might have benefits in individual non-motor symptoms subdomains assessing mood dysfunction/depression that were of a magnitude that would be subjectively meaningful to patients. These changes were not associated with changes in motor severity or other factors, suggesting exenatide may exert independent effects on mood dysfunction. These exploratory findings will contribute to the design of future trials that will confirm the extent of motor and non-motor symptom effects of exenatide in a larger cohort of patients.

\section{ACKNOWLEDGMENTS}

This study was funded by the Michael J. Fox Foundation for Parkinson's Research and the Cure Parkinson's Trust and coordinated by University College London's Comprehensive Clinical Trials Unit. This work was done partly at UCL and UCL Hospitals NHS Trust and was funded in part by the Department of Health National Institute for Health Research Biomedical Research Centres funding scheme. The trial was done at the Leonard Wolfson Experimental Neuroscience Centre (London, UK), a dedicated clinical trial research facility and part of the University College London (UCL) Institute of Neurology and the National Hospital for Neurology \& Neurosurgery. We thank the patients and their families who participated in the trial, and Vincenzo Libri and Rajeshree Khengar from the Leonard Wolfson Experimental Neuroscience Centre.

\section{CONFLICTS OF INTEREST}

PL has received honoraria from Medtronic and St. Jude Medical. TF has received honoraria from Profile Pharma, BIAL, AbbVie, Genus, Medtronic, and St Jude Medical. All other authors declare no competing interests.

\section{REFERENCES}

[1] Chaudhuri KR, Healy DG, Schapira AH (2006) Non-motor symptoms of Parkinson's disease: Diagnosis and management. Lancet Neurol 5, 235-245.

[2] Hely MA, Morris JGL, Reid WGJ, Trafficante R (2005) Sydney multicenter study of Parkinson's disease: NonL-dopa-responsive problems dominate at 15 years. Mov Disord 20, 190-199.

[3] Augustine EF, Pérez A, Dhall R, Umeh CC, Videnovic A, Cambi F, Wills A-MA, Elm JJ, Zweig RM, Shulman LM, Nance MA, Bainbridge J, Suchowersky O (2015) Sex differences in clinical features of early, treated Parkinson's disease. PLoS One 10, e0133002.

[4] Parkes DG, Mace KF, Trautmann ME (2013) Discovery and development of exenatide: The first antidiabetic agent to leverage the multiple benefits of the incretin hormone, GLP1. Expert Opin Drug Discov 8, 219-244.

[5] Athauda D, Maclagan K, Skene SS, Bajwa-Joseph M, Letchford D, Chowdhury K, Hibbert S, Budnik N, Zampedri L, Dickson J, Li Y, Aviles-Olmos I, Warner TT, Limousin P, Lees AJ, Greig NH, Tebbs S, Foltynie T (2017) Exenatide once weekly versus placebo in Parkinson's disease: A randomised, double-blind, placebo-controlled trial. Lancet 390, 664-1675.

[6] Chaudhuri KR, Martinez-Martin P, Schapira AHV, Stocchi F, Sethi K, Odin P, Brown RG, Koller W, Barone P, MacPhee G, Kelly L, Rabey M, MacMahon D, Thomas S, Ondo W, Rye D, Forbes A, Tluk S, Dhawan V, Bowron A, Williams 
AJ, Olanow CW (2006) International multicenter pilot study of the first comprehensive self-completed nonmotor symptoms questionnaire for Parkinson's disease: The NMSQuest study. Mov Disord 21, 916-923.

[7] Goldman JG, Postuma R (2014) Premotor and nonmotor features of Parkinson's disease. Curr Opin Neurol 27, 434441.

[8] Trenkwalder C, Kies B, Rudzinska M, Fine J, Nikl J, Honczarenko K, Dioszeghy P, Hill D, Anderson T, Myllyla V, Kassubek J, Steiger M, Zucconi M, Tolosa E, Poewe W, Surmann E, Whitesides J, Boroojerdi B, Chaudhuri KR, the RECOVER Study Group (2011) Rotigotine effects on early morning motor function and sleep in Parkinson's disease: A double-blind, randomized, placebo-controlled study (RECOVER). Mov. Disord 26, 90-99.

[9] Martinez-Martin P, Rodriguez-Blazquez C, Abe K, Bhattacharyya KB, Bloem BR, Carod-Artal FJ, Prakash R, Esselink RAJ, Falup-Pecurariu C, Gallardo M, Mir P, Naidu Y, Nicoletti A, Sethi K, Tsuboi Y, van Hilten JJ, Visser M, Zappia M, Chaudhuri KR (2009) International study on the psychometric attributes of the Non-Motor Symptoms Scale in Parkinson disease. Neurology 73, 1584-1591.

[10] Gallagher DA, Goetz CG, Stebbins G, Lees AJ, Schrag A (2012) Validation of the MDS-UPDRS Part I for nonmotor symptoms in Parkinson's disease. Mov Disord 27, 79-83.

[11] Martinez-Martin P, Chaudhuri KR, Rojo-Abuin JM, Rodriguez-Blazquez C, Alvarez-Sanchez M, Arakaki T, Bergareche-Yarza A, Chade A, Garretto N, Gershanik O, Kurtis MM, Martinez-Castrillo JC, Mendoza-Rodriguez A, Moore HP, Rodriguez-Violante M, Singer C, Tilley BC, Huang J, Stebbins GT, Goetz CG (2015) Assessing the nonmotor symptoms of Parkinson's disease: MDS-UPDRS and NMS Scale. Eur J Neurol 22, 37-43.

[12] Ketharanathan T, Hanwella R, Weerasundera R, de Silva VA (2016) Diagnostic validity and factor analysis of Montgomery-Asberg Depression Rating Scale in Parkinson disease population. J Geriatr Psychiatry Neurol 29, 115-119.

[13] Pirogovsky E, Schiehser DM, Litvan I, Obtera KM, Burke MM, Lessig SL, Song DD, Liu L, Filoteo JV (2014) The utility of the Mattis Dementia Rating Scale in Parkinson's disease mild cognitive impairment. Parkinsonism Relat Disord 20, 627-631.

[14] Li M, Yang HM, Luo DX, Chen JZ, Shi HJ (2016) Multidimensional analysis on Parkinson's disease questionnaire39 in Parkinson's patients treated with Bushen Huoxue Granule: A multicenter, randomized, double-blinded and placebo controlled trial. Complement Ther Med 29, 116120.

[15] Schrag A, Selai C, Jahanshahi M, Quinn NP (2000) The EQ5D-a generic quality of life measure-is a useful instrument to measure quality of life in patients with Parkinson's disease. J Neurol Neurosurg Psychiatry, 69, 67-73.

[16] Peto V, Jenkinson C, Fitzpatrick R (2001) Determining minimally important differences for the PDQ-39 Parkinson's disease questionnaire. Age Ageing 30, 299-302.
[17] Chaudhuri KR, Martinez-Martin P, Brown RG, Sethi K, Stocchi F, Odin P, Ondo W, Abe K, MacPhee G, MacMahon D, Barone P, Rabey M, Forbes A, Breen K, Tluk S, Naidu Y, Olanow W, Williams AJ, Thomas S, Rye D, Tsuboi Y, Hand A, Schapira AHV (2007) The metric properties of a novel non-motor symptoms scale for Parkinson's disease: Results from an international pilot study. Mov Disord 22, 1901-1911.

[18] Aviles-Olmos I, Dickson J, Kefalopoulou Z, Djamshidian A, Ell P, Soderlund T, Whitton P, Wyse R, Isaacs T, Lees A, Limousin P, Foltynie T (2013) Exenatide and the treatment of patients with Parkinson's disease, J Clin Invest 123, 27302736.

[19] Aviles-Olmos I, Dickson J, Kefalopoulou Z, Djamshidian A, Kahan J, Fmedsci PE, Whitton P, Wyse R, Isaacs T, Lees A, Limousin P, Foltynie T (2014) Motor and cognitive advantages persist 12 months after exenatide exposure in Parkinson's disease. J Parkinsons Dis 4, 337-344.

[20] Marsh L (2013) Depression and Parkinson's disease: current knowledge. Curr Neurol Neurosci Rep 13, 409.

[21] Anderberg RH, Richard JE, Hansson C, Nissbrandt H, Bergquist F, Skibicka KP (2016) GLP-1 is both anxiogenic and antidepressant; divergent effects of acute and chronic GLP-1 on emotionality. Psychoneuroendocrinology 65, 54-66.

[22] Ventorp F, Bay-Richter C, Nagendra AS, Janelidze S, Matsson VS, Lipton J, Nordström U, Westrin Åsa, Brundin P, Brundin L (2017) Exendin-4 treatment improves LPS-induced depressive-like behavior without affecting pro-inflammatory cytokines. J Parkinsons Dis 7, 263-273.

[23] Storch A, Schneider CB, Klingelhöfer L, Odin P, Fuchs G, Jost WH, Martinez-Martin P, Koch R, Reichmann H, Chaudhuri KR, Ebersbach G (2015) Quantitative assessment of non-motor fluctuations in Parkinson's disease using the Non-Motor Symptoms Scale (NMSS). J Neural Transm 122, 1673-1684.

[24] Pagonabarraga J, Kulisevsky J, Strafella AP, Krack P (2015) Apathy in Parkinson's disease: Clinical features, neural substrates, diagnosis, and treatment. Lancet Neurol 14, 518531.

[25] Martinez-Martin P, Reddy P, Antonini A, Henriksen T, Katzenschlager R, Odin P, Todorova A, Naidu Y, Tluk S, Chandiramani C, Martin A, Chaudhuri KR (2011) Chronic subcutaneous infusion therapy with apomorphine in advanced Parkinson's disease compared to conventional therapy: A real life study of non motor effect. J Parkinsons Dis 1, 197-203.

[26] Martinez-Martin P, Reddy P, Katzenschlager R, Antonini A, Todorova A, Odin P, Henriksen T, Martin A, Calandrella D, Rizos A, Bryndum N, Glad A, Dafsari HS, Timmermann L, Ebersbach G, Kramberger MG, Samuel M, Wenzel K, Tomantschger V, Storch A, Reichmann H, Pirtosek Z, Trost M, Svenningsson P, Palhagen S, Volkmann J, Chaudhuri KR (2015) EuroInf: A multicenter comparative observational study of apomorphine and levodopa infusion in Parkinson's disease. Mov Disord 30, 510-516. 